\title{
The role of FIS in the Rcd checkpoint and stable maintenance of plasmid ColE1
}

\author{
I. K. Blabyt and D. K. Summers \\ Department of Genetics, University of Cambridge, Downing Street, Cambridge CB2 3EH, UK
}

Correspondence

D. K. Summers

dks11@cam.ac.uk

\begin{abstract}
Escherichia coli plasmid ColE1 lacks active partitioning, and copies are distributed randomly to daughter cells at division. The plasmid is maintained stably in the bacterial population as long as its copy number remains high. The accumulation of plasmid dimers and higher multimers depresses copy number, and is an important cause of multicopy plasmid instability. ColE1 dimers are restored to the monomeric state by site-specific recombination, which requires the hostencoded proteins XerCD, ArgR and PepA acting at the plasmid cer site. In addition, a 70 nt RNA expressed from the cer site of plasmid dimers delays the division of dimer-containing cells. Here, we report that the global regulator FIS binds to cer in a sequence-specific manner, close to the Rcd promoter $\left(\mathrm{P}_{c e r}\right)$. FIS is not required for plasmid dimer resolution, but is essential for repression of $\mathrm{P}_{c e r}$ in plasmid monomers. Repression also requires the XerCD recombinase, but not ArgR or PepA. We propose a model for monomer-dimer control of $\mathrm{P}_{\text {cer }}$ in which the promoter is repressed in plasmid monomers by the concerted action of FIS and XerCD. Rcd transcription is triggered in plasmid dimers by the lifting of XerCD-mediated repression in the synaptic complex.
\end{abstract}

Received 2 April 2009
Revised 7 May 2009

Accepted 11 May 2009

\section{INTRODUCTION}

The formation of multicopy plasmid dimers, and the associated reduction in copy number, is an important cause of instability (Summers \& Sherratt, 1984). Even when the rate of de novo dimer formation is low, the ability of dimers to out-replicate monomers means that they accumulate rapidly and clonally (the so-called dimer catastrophe). This process creates a subpopulation of cells in the culture from which plasmid-free variants arise at high frequency (Summers et al., 1993). The Escherichia coli plasmid ColE1 combats the threat of the dimer catastrophe by resolving dimers to monomers via site-specific recombination at the cer site (Fig. 1; Summers \& Sherratt, 1984). Multimer resolution requires four chromosome-encoded proteins: XerC and XerD (which together form the heterodimeric recombinase), and accessory proteins ArgR and PepA (Blakely et al., 1993; Colloms et al., 1990; Stirling et al., 1988, 1989). ArgR and PepA impose topological constraint on the system so that dimer resolution predominates over dimer formation (Guhathakurta et al., 1996). Models have been proposed for the structure of nucleoprotein complexes that form at individual cer sites (Hodgman et al., 1998), and for the synaptic complex that forms during dimer resolution (Hodgman et al., 1998; Strater et al., 1999).

†Present address: Department of Microbiology and Cell Science, PO Box 110700, University of Florida, Gainesville, FL 32611-0700, USA.

Abbreviation: EMSA, electrophoretic mobility shift assay. cer-mediated dimer resolution is necessary, but not sufficient, to ensure plasmid stability. Rcd, a small regulatory RNA encoded within cer, is also required. Rcd is transcribed from the $\mathrm{P}_{c e r}$ promoter in plasmid dimers, but not in monomers, and is a key component of the checkpoint that delays the division of dimer- or multimercontaining cells (Balding et al., 2006; Patient \& Summers, 1993). Binding of Rcd to its target tryptophanase reduces the $K_{\mathrm{m}}$ of this enzyme for tryptophan, and stimulates the production of indole (Chant \& Summers, 2007). The elevation of intracellular indole is thought to be responsible for delaying cell division, although the mechanism is unknown.

$\mathrm{P}_{c e r}$ exhibits monomer-dimer control; it is inactive in plasmid monomers, but active in dimers and higher multimers. A characteristic feature of the promoter is that the spacer between the -35 and -10 boxes is $15 \mathrm{bp}$, rather than the E. coli consensus of 17 bp (Fig. 1). Insertion of $2 \mathrm{bp}$ into the spacer (corresponding to a change in the angular separation of the -10 and -35 boxes of approximately $70^{\circ}$ ) results in a 10 - to 20 -fold increase in transcription. This has led to the proposal that twisting of the promoter in the synaptic complex formed by XerCD, ArgR, PepA and the two cer sites in a plasmid dimer realigns the -35 and -10 regions, and activates $\mathrm{Rcd}$ transcription (Chatwin \& Summers, 2001).

In this report, we demonstrate that a fifth chromosomeencoded protein, FIS, binds to cer in a sequence-specific manner, and show that mutations that disrupt binding 
GTGAAACCAT GAAAATG GC AGTTCAGTG GATTAAGT

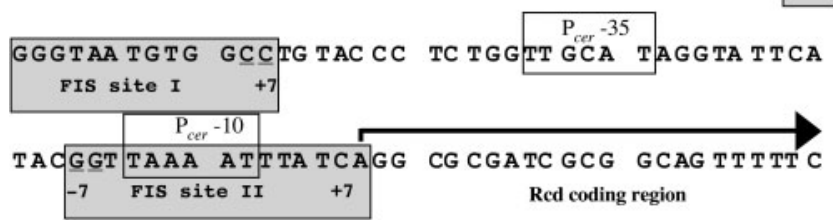

Fig. 1. DNA sequence surrounding the $P_{\text {cer }}$ promoter of ColE1 cer. The -10 and -35 sequences of the promoter are enclosed in open boxes. Two putative FIS-binding sites are enclosed in grey boxes. The start of the Rcd coding region is indicated by an arrow.

cause plasmid instability. FIS appears to have little or no influence on cer-mediated recombination, but, along with $\mathrm{XerC}$ and XerD, it is required for proper regulation of the $\mathrm{P}_{c e r}$ promoter. On the basis of these observations, we propose a model for monomer-dimer control of $\mathrm{P}_{c e r}$ in which FIS and XerCD together repress $\mathrm{P}_{c e r}$ in plasmid monomers. Rcd transcription is triggered from dimers when formation of the synaptic complex removes XerCD from one of the two promoters.

\section{METHODS}

Bacterial strains and plasmids. Strain DS941 is E. coli K-12 AB1157 recF lacl ${ }^{q}$ lacZLM15 (Summers \& Sherratt, 1984), and is deficient in plasmid recombination. JC8679 is AB1157 recB21 recC22 sbcA23 his328 (Gillen et al., 1981), and is hyper-recombinogenic for plasmids. Fis $^{-}$derivatives were constructed by Dr P. Oliver by $\mathrm{P} 1$ transduction of the fis-767 allele from RJ2452 (Johnson et al., 1988) into the relevant background. XL1-Blue is described by Bullock et al. (1987). Cultures of bacteria were grown routinely at $37{ }^{\circ} \mathrm{C}$ in L-broth or on L-agar (Kennedy, 1971). Antibiotics were added to media when appropriate (carbenicillin, $100 \mu \mathrm{g} \mathrm{ml}^{-1}$; chloramphenicol, $100 \mu \mathrm{g} \mathrm{ml}^{-1}$ ).

Plasmid pKS490 is a derivative of pUC8 containing cer as a TaqI-HpaII fragment inserted into the polylinker AccI site (Summers \& Sherratt, 1984; Vieira \& Messing, 1982). pCA24N-FIS is a derivative of pCA24N (Kitagawa et al., 2005), and it contains the fis ORF with an $\mathrm{N}$-terminal $6 \times$ His tag, and a C-terminal GFP fusion. pCA24N-FIS $\triangle$ GFP was derived from pCA24N-FIS by digestion with NotI, and religation to delete the GFP-coding region. Plasmid stability in serial batch culture was assayed as described by Chant \& Summers (2007).

Electrophoretic mobility shift assay. His-tagged FIS was produced from XL1-Blue containing pCA24N-FIS $\Delta$ GFP in 2YT medium (16 g tryptone $\mathrm{l}^{-1}, 10 \mathrm{~g}$ yeast extract $\mathrm{l}^{-1}$ and $5 \mathrm{~g} \mathrm{NaCl}^{-1}$ ), and cultured initially at $37^{\circ} \mathrm{C}$. Protein production was induced with IPTG $(0.1-$ $1 \mathrm{mM})$ at $\mathrm{OD}_{600} 0.6$, and incubation was continued for $2-3 \mathrm{~h}$ at room temperature. Cells were lysed with lysozyme $\left(1 \mathrm{mg} \mathrm{ml}^{-1}\right)$. Histagged protein was purified using nickel resin columns (Amersham Biosciences), and yield and purity were monitored by SDS-PAGE. DNA substrates for the electrophoretic mobility shift assay (EMSA) were generated by PCR using the relevant plasmid as a template. For each substrate, a second round of PCR was performed to minimize the concentration of contaminating plasmid DNA. EMSA reactions comprised the following. DNA of a constant concentration (typically
$1 \mu \mathrm{g}$ ) was added to $4 \mu \mathrm{l}$ binding buffer [20 mM Tris/HCl ( $\mathrm{pH} 8.0$ ), $0.4 \mathrm{M}$ EDTA, $0.1 \mathrm{mM}$ DTT, $50 \mathrm{mM} \mathrm{NaCl}, 1 \mathrm{mM} \mathrm{MgCl}_{2}$, and $12.5 \%$ (w/v) glycerol], $1 \mu \mathrm{l}$ pdIdC $\left(1 \mu \mathrm{g} \mathrm{ml}^{-1}\right)$ and purified FIS protein. Reactions were made up to a final volume of $30 \mu \mathrm{l}$ with sterile distilled water. The mixture was centrifuged briefly, and incubated at room temperature for $1 \mathrm{~h}$. A $4 \mu \mathrm{l}$ volume of $60 \%(\mathrm{v} / \mathrm{v})$ glycerol was added to each reaction before loading onto a $7 \%$ polyacrylamide gel. Non-specific competitor DNA ( $80 \mathrm{bp}$ ) was generated from the cer site by PCR using primers CGCGATCGCGCGAGTTTTTC and GCTAAAACGCGTTCAGCGCG. The amplified fragment covers the Rcd coding region of $c e r$, but does not include the adjacent putative FIS-binding sites. Competitor DNA (42 bp), containing a FIS consensus sequence (underlined), was as follows: AATTCCCGAGCTGGCCAAACTTTGACCCTAGATCTGGGGATC.

Site-directed mutagenesis by overlap PCR. Defined base changes were introduced into putative FIS-binding sites by overlap PCR (Higuchi et al., 1988; Ho et al., 1989)

RNA assay by quantitative real-time PCR. RNA was prepared using Trizol (Invitrogen), and contaminating DNA was digested by DNase I treatment (Ambion), according to the manufacturer's directions. cDNA was synthesized from the RNA using enhanced avian reverse transcriptase (Sigma), according to the manufacturer's protocol. The cDNA was amplified by quantitative real-time PCR using a Bio-Rad IQ5 instrument (the product was stained using SYBR green; Bio-Rad). The threshold cycle $\left(C_{\mathrm{T}}\right)$ data were interpreted using the Livak method (Livak \& Schmittgen, 2001). Control experiments were performed to confirm that no significant signal was obtained when the reverse transcription step was omitted.

\section{RESULTS}

\section{FIS binds to cer in a sequence-specific manner}

XerC, XerD, ArgR and PepA are both necessary and sufficient for cer-mediated dimer recombination in vitro (Colloms et al., 1996). However, additional proteins may be required for regulation of $\mathrm{P}_{c e r}$ and the Rcd checkpoint, even if they have no direct role in Xer-cer recombination. A possible candidate is FIS. pUC-based plasmids are unstable in a fis mutant host, and sequence analysis has identified two putative binding sites for this global regulator protein that are close to $\mathrm{P}_{c e r}$ (Balding et al., 2006; Fig. 1).

To discover whether FIS binds to cer in vitro, a series of EMSAs was performed using N-terminal His-tagged FIS produced from pCA24N-FIS $\Delta$ GFP. To ensure that the biological activity of the protein was unaffected by the His tag, we confirmed that production of the His-tagged protein complemented multicopy plasmid instability in a Fis $^{-}$host (data not shown). The DNA substrate was a 364 bp fragment containing the whole cer site, which was generated from plasmid pKS490 using M13 universal forward and reverse primers. A band shift (s1) was seen initially at $0.33 \mu \mathrm{M}$ FIS, and a second (s2) was visible at $1.2 \mu \mathrm{M}$ (Fig. 2a). At $3.3 \mu \mathrm{M}$ FIS, the DNA substrate band was weaker than either of the shifted bands. Further experiments were performed to explore whether the shifts were due to sequence-specific binding by FIS. In the first experiment (Fig. 2b), the FIS concentration was main- 
(a)

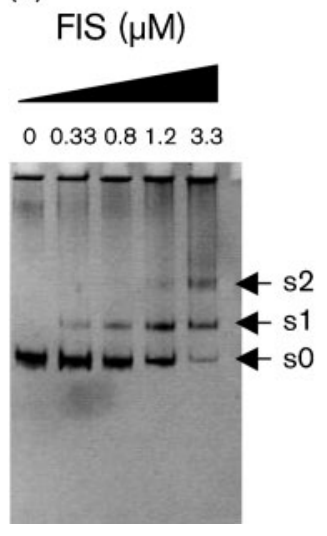

(b)

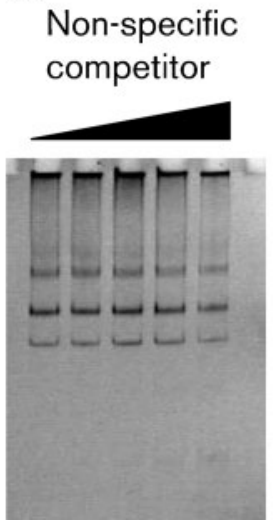

(c)

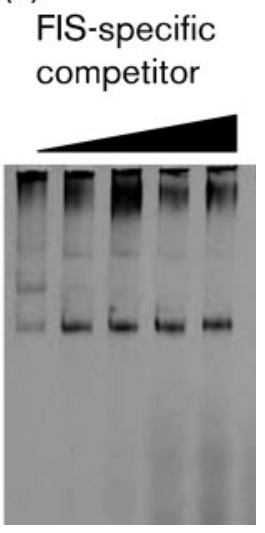

Fig. 2. Mobility shift assay of FIS binding to cer site DNA. (a) A 364 bp DNA substrate (s0) was incubated with FIS at concentrations up to a maximum of $3.3 \mu \mathrm{M}$. (b) DNA substrate incubated with $3.3 \mu \mathrm{M}$ FIS was challenged with an increasing concentration of competitor DNA lacking a FIS-binding site (maximum 1.5fold molar excess, 0.3-fold by weight, over the cer DNA). (c) DNA substrate incubated with 3.3 $\mu \mathrm{M}$ FIS was challenged with an increasing concentration of competitor DNA containing a FIS-binding site (maximum 1.5-fold molar excess, 0.2-fold by weight, over the cer DNA). tained at $3.3 \mu \mathrm{M}$, and the complex was challenged with an increasing concentration of an $80 \mathrm{bp}$ control fragment that lacked a FIS-binding site. This had no effect on the distribution of the cer substrate between shifted and nonshifted bands. Next, the complex was challenged with an increasing concentration of a 42 bp DNA fragment containing a sequence closely matching the consensus FIS-binding site (Finkel \& Johnson, 1992) (Fig. 2c). Band s1 was reduced significantly even at the lowest concentration of competitor, and at the maximum competitor concentration shifted bands were almost completely absent.

To identify where FIS binds, a series of mutations was introduced into the two putative binding sites (Fig. 1). In site I, mutations were introduced at positions -6 and -7 (GG to $\mathrm{AA})$, and/or at +6 and +7 (CC to AA). In site II, mutations were introduced at -6 and -7 (GG to TT). The importance of the \pm 7 positions in the FIS-binding site sequence logo (Hengen et al., 1997) suggests that changes should have a major effect on binding. EMSAs were performed on cer sites containing combinations of these mutations (Fig. 3). In each case, the EMSA profile of the mutant site was compared, on the same gel and with the same FIS protein preparation, with wild-type cer. Mutation of the left $(-6 /-7)$ or right $(+6 /$ +7 ) end of site I, or a combination of both, had very little effect upon the EMSA profile (Fig. 3a-c). However, mutation of the left end of site II led to the complete disappearance of band s1 (Fig. 3d). A very similar pattern was seen when left- and right-end mutations of site I were combined with the site II mutations (Fig. 3e).

\section{FIS-binding-site mutations cause plasmid instability}

We assayed the effect of mutations in the putative FISbinding sites on plasmid stability over 100 generations of non-selective growth by using the method of Chant \& Summers (2007). The stability of pKS490 (pUC8cer) and derivatives with various FIS-binding site mutations (pIKB5, pIKB6, pIKB7, pIKB8 and pIKB9; Fig. 3) was assayed in the hyper-recombinogenic strain JC8679. pKS490 was retained by $100 \%$ of cells after 100 generations of non-selective growth. However, all of the mutant plasmids showed reduced stability, with only $40-60 \%$ of cells retaining the plasmids after 100 generations (Table 1). Surprisingly, in the light of the EMSA results, mutations in either FIS-binding site (or in both binding sites) resulted in similar instability. The experiment was repeated using a fis mutant derivative of JC8679. This time all of the plasmids were highly unstable (Table 1), and pKS490 was no longer distinguishable from its mutant derivatives; this is consistent with altered binding of FIS to cer being responsible for the observed plasmid instability.

The instability of plasmids with mutated FIS-binding sites could be due to failure of the Rcd checkpoint, or to inefficient dimer resolution. The recombination proficiency of the mutant sites was tested by introducing dimers of pKS490 and the series of mutant derivatives into DS941, and assessing the multimeric state of plasmids in the transformants. All transformants contained plasmid monomers (data not shown). Furthermore, efficient dimer resolution was also observed when dimers were transformed into a Fis ${ }^{-}$derivative of DS941. Thus FIS binding to $\mathrm{cer}$ is not required for effective dimer resolution.

Since dimer resolution was unaffected, we explored the possibility that FIS is required for the proper operation of the Rcd checkpoint. Although FIS was discovered originally through its role in site-specific recombination, it participates in a variety of other DNA transactions, including replication and the regulation of transcription (Finkel \& Johnson, 1992). Given the overlap of FIS site II with the -10 sequence of $\mathrm{P}_{c e r}$, a role in the regulation of $\mathrm{Rcd}$ transcription seemed plausible. Total RNA was prepared from cultures of DS941, DS941fis, JC8679 and JC8679fis, each containing the cer plasmid pKS490. RNA was reverse transcribed using primers complementary to $\operatorname{Rcd}$ and $\beta$ lactamase (bla) mRNA. The products of reverse transcription were quantified by real-time PCR. Since bla is transcribed constitutively (Nordström et al., 1972), the concentration of bla mRNA is proportional to the plasmid copy number (strictly to the number of plasmid monomer equivalents). The ratio of Rcd to bla mRNA therefore 
(a)
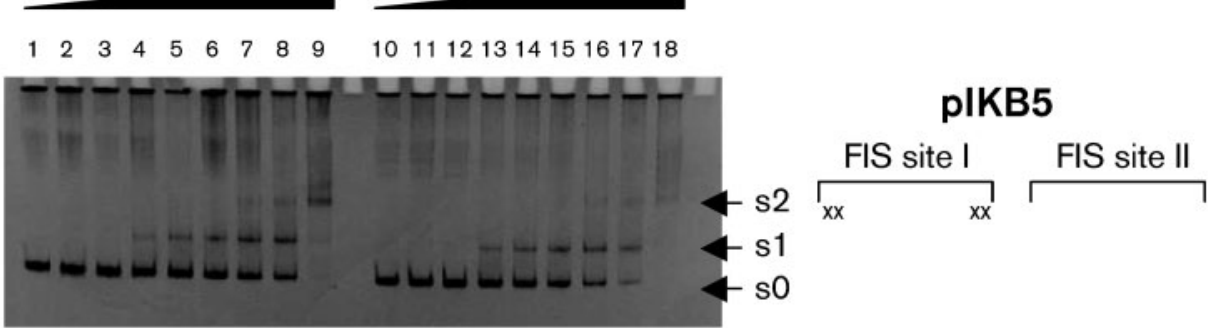

(b)

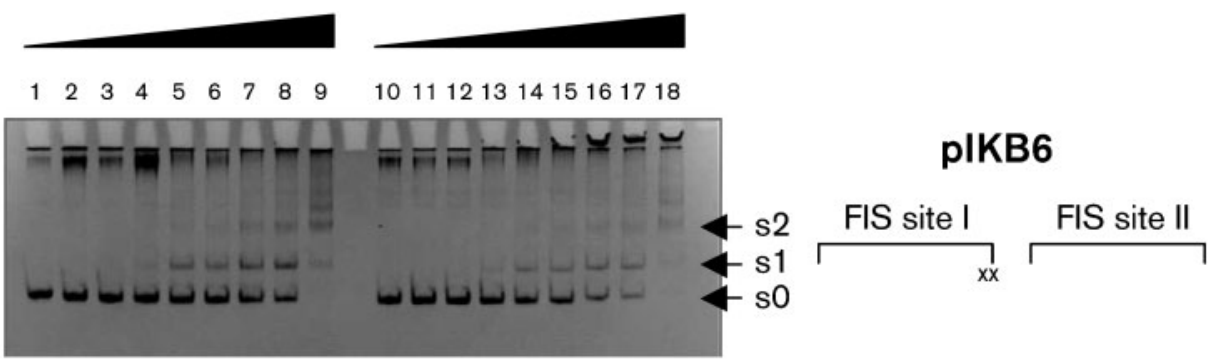

(c)
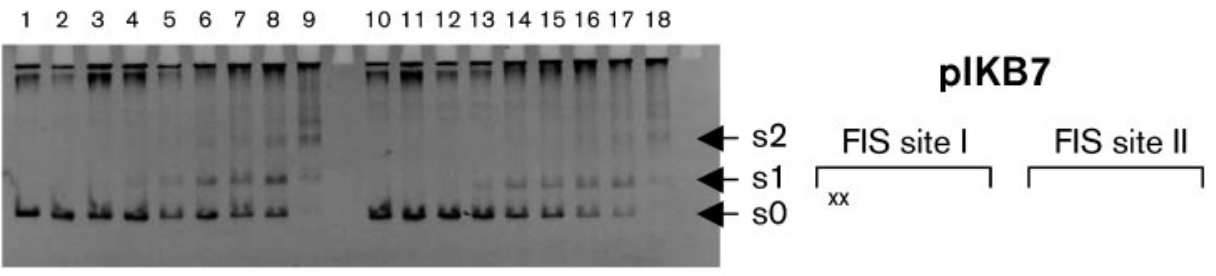

(d)

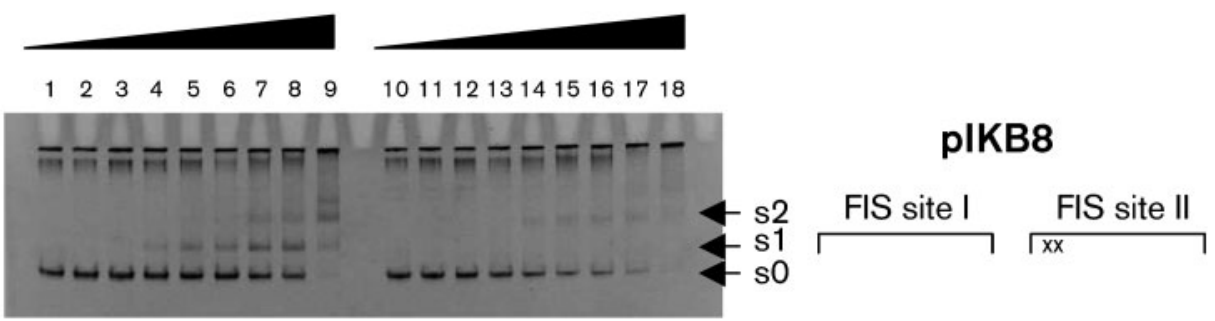

(e)
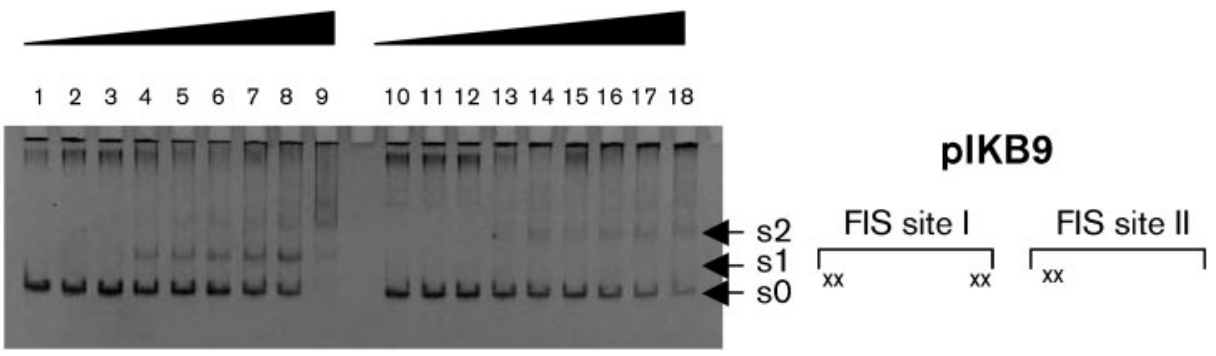

Fig. 3. EMSA comparing binding of FIS to wild-type and mutant cer sites. Wild-type cer substrate (lanes 1-9;0, 1.6 and $16 \mathrm{nM}, 0.3,0.6,0.8,1.2,1.6$ and $3.3 \mu \mathrm{M}$, respectively) was compared with substrate derived from mutant cer sites (lanes 11$19 ; 0,1.6$ and $16 \mathrm{nM}, 0.3,0.6,0.8,1.2,1.6$ and $3.3 \mu \mathrm{M}$, respectively). Mutant substrates were amplified from plasmids plKB5 (a), plKB6 (b), plKB7 (c), pIKB8 (d) and pIKB9 (e). A schematic shown beside each gel image indicates how each FIS-binding site has been mutated (mutations indicated with $\mathrm{x}$ ). 
Table 1. Stabilities of plasmids with mutant FIS-binding sites

\begin{tabular}{|lccc|}
\hline Strain and plasmid & $\begin{array}{c}\text { FIS-binding site } \\
\text { mutated }\end{array}$ & $\begin{array}{c}\text { JC8679, plasmid- } \\
\text { containing cells (\%) }\end{array}$ & $\begin{array}{c}\text { JC8679fis, plasmid- } \\
\text { containing cells (\%) }\end{array}$ \\
\hline JC8679 pKS490 & Wild-type cer & $100 \pm 0$ & $6 \pm 4$ \\
JC8679 pIKB5 & I & $40 \pm 7$ & $7 \pm 9$ \\
JC8679 pIKB6 & I & $45 \pm 7$ & $5 \pm 10$ \\
JC8679 pIKB7 & I & $56 \pm 12$ & $4 \pm 6$ \\
JC8679 pIKB8 & II & $48 \pm 7$ & $3 \pm 4$ \\
JC8679 pIKB9 & I II & $62 \pm 10$ & $23 \pm 17$ \\
\hline
\end{tabular}

${ }^{*}$ Data are the means \pm SD of at least five independent experiments. Plasmid content was assayed after 100 generations of growth in non-selective broth.

provides a measure of Rcd produced per plasmid. This normalization is important because the copy number of pUC8 derivatives is depressed by approximately $50 \%$ in the absence of FIS (our unpublished data). No Rcd was detected in plasmid-free controls. Rcd concentrations in plasmid-containing cells were expressed relative to the basal level in DS941 pKS490 where the plasmid is predominantly monomeric (Table 2). Comparison of transcription from pKS490 in DS941 and JC8679 showed a 12.5-fold increase in Rcd production associated with the multimerization of the plasmid in JC8679. A comparison of Rcd production in DS941 and DS941 fis revealed a 10fold increase in the latter, implying that FIS acts as a repressor of $\mathrm{P}_{c e r}$ in plasmid monomers.

If the production of Rcd from pKS490 monomers in the Fis $^{-}$host is due to the absence of FIS from binding sites upstream of $\mathrm{P}_{c e r}$, mutations that affect FIS binding to these sites should also de-repress transcription. We introduced pKS490 and the series of mutant derivatives containing mutations in FIS sites I and II (pIKB5, pIKB6, pIKB7, pIKB8 and pIKB9) into DS941. Total RNA was isolated and Rcd per plasmid was quantified by real-time PCR (Table 3). As a control to identify any FIS-independent effects of the mutations on transcription, Rcd production was also assayed in DS941fis. Plasmids pIKB5, pIKB6 and pIKB7 (which are mutated in site I) showed a three- to

Table 2. Relative Rcd production per plasmid from a wild-type cer site

\begin{tabular}{|lc|}
\hline Strain & Rcd per $^{\text {plasmid }}{ }^{*}$ \\
\hline DS941 pKS490 & 1.00 \\
DS941 fis pKS490 & $9.5 \pm 1.5$ \\
JC8679 pKS490 & $12.5 \pm 1.6$ \\
JC8679fis pKS490 & $19.6 \pm 4.5$ \\
DS941 xerC pKS490 & $74 \pm 13$ \\
DS941 xerD pKS490 & $98 \pm 18$ \\
DS941 argR pKS490 & $1.2 \pm 0.5$ \\
DS941 pepA pKS490 & $1.6 \pm 0.4$ \\
\hline
\end{tabular}

${ }^{\star}$ Data are the means \pm SD of three independent experiments. fivefold elevation of Rcd. Plasmids pIKB8 (which is mutated in site II alone) and pIKB9 (mutated in both sites I and II) showed an approximately eightfold elevation of Rcd. When the experiment was performed in a Fis ${ }^{-}$ host, none of the mutations caused a significant increase in Rcd production over that seen for pKS490 monomers.

Our data from EMSA, and from assays of transcription in vivo, suggest strongly that binding of FIS to site II (which overlaps the -10 sequence of $\mathrm{P}_{c e r}$ ) is necessary for repression of $\mathrm{P}_{c e r}$ in plasmid monomers. The transcription assays suggest that binding at site I also has a role. Is it possible that FIS repression in plasmid monomers, and the lifting of this repression in dimers, might provide a complete explanation for the monomer-dimer control of $\mathrm{P}_{c e r}$ ? If the activation of $\mathrm{P}_{c e r}$ in dimers is due solely to the lifting of FIS-mediated repression, the same level of transcription should be seen in DS941 fis pKS490 and JC8679fis pKS490. In fact $\mathrm{P}_{c e r}$ activity is twofold higher in the multimer-containing strain (Table 2), indicating that additional factors are involved in $\mathrm{P}_{c e r}$ regulation. To discover whether any of the other proteins that assemble at cer (ArgR, PepA XerC and XerD) contribute to $\mathrm{P}_{c e r}$ repression, we assayed Rcd production from plasmid monomers in mutant strains lacking each of these proteins in turn (Table 2). The absence of ArgR or PepA had no effect on transcription, but, in the absence of XerC or XerD, $\mathrm{P}_{c e r}$ activity increased by 74 - and 98 -fold, respectively. It therefore appears that both FIS and XerCD act as repressors of $\mathrm{P}_{c e r}$.

\section{DISCUSSION}

It is clear that FIS must be added to the list of participants in the nucleoprotein complex at ColE1 cer. FIS is not required for the conversion of plasmid dimers to monomers, but it is required for the repression of $\mathrm{P}_{c e r}$ in plasmid monomers, and is therefore essential for the proper operation of the Rcd checkpoint.

Sequence analysis of cer (Balding et al., 2006) identified two possible FIS-binding sites. However, our EMSA results provide evidence for binding to only one of these sites. (Although mutations in site II caused a substantial 
Table 3. Relative Rcd production per plasmid from cer sites with mutant FIS-binding sites

\begin{tabular}{|lccc|}
\hline Strain & Rcd per plasmid $^{*}$ & Strain & Rcd per $^{\text {plasmid }}{ }^{*}$ \\
\hline DS941 pKS490 & 1.0 & DS941 fis pKS490 & 1.0 \\
DS941 pIKB5 & $5.4 \pm 0.5$ & DS941 fis pIKB5 & $0.9 \pm 0.4$ \\
DS941 pIKB6 & $3.1 \pm 0.4$ & DS941fis pIKB6 & $1.2 \pm 0.5$ \\
DS941 pIKB7 & $4.6 \pm 0.5$ & DS941fis pIKB7 & $1.1 \pm 0.3$ \\
DS941 pIKB8 & $8.3 \pm 0.4$ & DS941 fis pIKB8 & $1.0 \pm 0.2$ \\
DS941 pIKB9 & $7.8 \pm 1.0$ & DS941fis pIKB9 & $1.1 \pm 0.3$ \\
\hline
\end{tabular}

${ }^{\star}$ Data are the means \pm SD of three independent experiments.

reduction in retarded bands in the EMSA, mutations in site I had very little effect.) A different result was obtained when we assayed the effect of the same binding-site mutations on Rcd production in vivo. We found that mutation of either or both sites induced Rcd production from plasmid monomers. This suggests that FIS may bind to both sites in vivo. It is possible that binding to site I requires supercoiled DNA (the EMSA substrate being relaxed, linear DNA). An alternative interpretation might be that the introduction of mutations into site I induces Rcd transcription in vivo by an effect on the binding of another protein (e.g. ArgR). However, our observation that Rcd production from mutant and wild-type plasmids was indistinguishable in a Fis ${ }^{-}$host is consistent with the effect being due to altered FIS binding. Site II overlaps the -10 region of $\mathrm{P}_{c e r}$ (Fig. 1), so it is easy to imagine how FIS bound here could repress $\mathrm{P}_{c e r}$ transcription. FIS site I is 13 bp upstream of the -35 region of $\mathrm{P}_{c e r}$, and, although a direct effect upon the promoter cannot be ruled out, it is possible that the role of site I is to maintain a high local concentration of FIS in the vicinity of site II (a similar idea has been proposed for the lac operon; Muller-Hill, 2006). Alternatively, there might be cooperativity between FIS bound at the two sites, with FIS binding at site II being a pre-requisite for binding at site I.

It is evident from our data (Table 2) that full repression of $\mathrm{P}_{c e r}$ in plasmid monomers requires both FIS and the XerCD recombinase. The XerCD-binding site lies approximately
$100 \mathrm{bp}$ downstream of $\mathrm{P}_{c e n}$, so the recombinase exerts repression from a distance. In our model of the nucleoprotein complexes involved in ColE1 dimer resolution (Hodgman et al., 1998), the cer site DNA wraps twice around a core of ArgR and PepA protein hexamers (Fig. 4a). We note that wrapping of the DNA brings XerCD very close to $\mathrm{P}_{c e n}$ suggesting that the recombinase may exert repression by blocking access of RNA polymerase to the promoter.

The constitutive activation of $\mathrm{P}_{c e r}$ in the absence of FIS or XerCD has caused us to revisit the question of how the promoter discriminates between plasmid monomers and dimers. We suggested previously (Chatwin \& Summers, 2001) that $\mathrm{P}_{c e r}$ is inactive in monomers because of the suboptimal alignment of the -35 and -10 boxes. Promoter activation in dimers was thought to result from the restoration of an optimal alignment by twisting or bending of the promoter in the synaptic complex between two cer sites in a dimer. Our observation that the removal of FIS or XerCD is sufficient to induce transcription from $\mathrm{P}_{c e r}$ in plasmid monomers refutes this idea. If realignment of the -35 and -10 of $\mathrm{P}_{c e r}$ is necessary for promoter activity, this must already have happened during formation of the monomer nucleoprotein complex. Therefore, promoter realignment cannot be responsible for $\mathrm{P}_{c e r}$ activation in dimers. An alternative mechanism is suggested by a closer examination of our model of Xer-cer synaptic complex formation (Hodgman et al., 1998). A simplified representation of this model is shown in Fig. 4. (a)

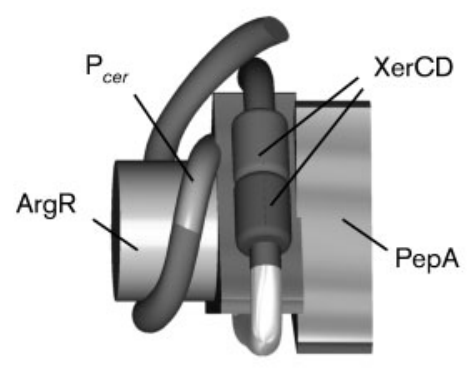

(b)

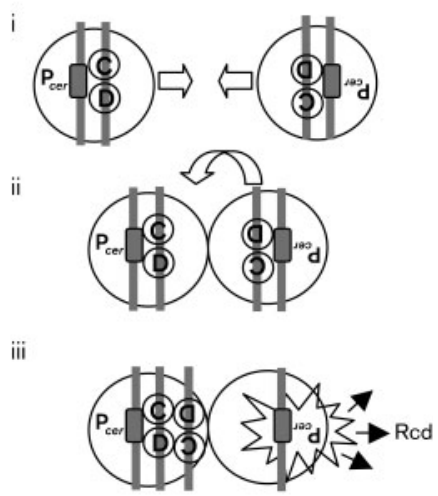

Fig. 4. A model for monomer-dimer control of the $\mathrm{P}_{\text {cer }}$ promoter. (a) The proposed structure of the single-site nucleoprotein complex at cer (Hodgman et al., 1998). (b) Juxtaposition of two single-site complexes (i) forms the paired complex (ii) in which the two XerCD heterodimers are well separated and each is in close proximity to a $\mathrm{P}_{\text {cer }}$ promoter. Isomerization (the 'gear shift') forms the synaptic complex (iii), and removes the right-hand XerCD from the vicinity of the $\mathrm{P}_{c e r}$ promoter, inducing transcription of Rcd. 
We propose that in plasmid monomers a single-site complex assembles at $\operatorname{cer}$ (Fig. 4a). Following dimer formation, two single-site complexes associate to form a paired-site complex (Fig. 4b). However, the XerCD recombinases are far apart in this complex, and are unable to recombine until the gear shift isomerization has brought them into close proximity in the synaptic complex. This isomerization removes XerCD from one of the two $\mathrm{P}_{c e r}$ promoters in the synaptic complex, and, our transcription data from $x e r C$ and $x e r D$ mutants (Table 2) suggest, should strongly induce the promoter. We imagine that FIS would remain bound to both promoters during synaptic complex formation, but would not prevent activation because FIS is unable to repress $\mathrm{P}_{c e r}$ in the absence of XerCD (note that in Table 2 the $x e r C / D$ mutant strains are Fis $^{+}$). Thus, in this model, the role of FIS is to ensure high fidelity repression of $\mathrm{P}_{c e r}$ in plasmid monomers.

Although we no longer believe that the suboptimal spacer of $\mathrm{P}_{c e r}$ is central to monomer-dimer control of the promoter, it may not be without functional significance. It is essential that $\mathrm{P}_{c e r}$ is repressed at all times when the plasmid is monomeric. During replication of a plasmid monomer, any proteins assembled at cer will be transiently displaced, and the temporary loss of FIS and XerCD might be expected to cause a brief burst of transcription from $\mathrm{P}_{c e r}$. However, the short spacer of the naked promoter ensures that it presents an unattractive substrate for RNA polymerase (Chatwin \& Summers, 2001), thus minimizing unwanted production of Rcd.

\section{ACKNOWLEDGEMENTS}

I. K. B. was the recipient of a $\mathrm{PhD}$ studentship from the Medical Research Council (UK). We thank Hywel Griffiths for the production of Fig. 4(a).

\section{REFERENCES}

Balding, C., Blaby, I. \& Summers, D. (2006). A mutational analysis of the ColE1-encoded cell cycle regulator Rcd confirms its role in plasmid stability. Plasmid 56, 68-73.

Blakely, G., May, G., McCulloch, R., Arciszewska, L. K., Burke, M., Lovett, S. T. \& Sherratt, D. J. (1993). 2 related recombinases are required for site-specific recombination at dif and cer in Escherichia coli K12. Cell 75, 351-361.

Bullock, W. O., Fernandez, J. M. \& Short, J. M. (1987). XL1-Blue: a high-efficiency plasmid transforming recA Escherichia coli strain with $\beta$-galactosidase selection. Biotechniques 5, 376-378.

Chant, E. L. \& Summers, D. K. (2007). Indole signalling contributes to the stable maintenance of Escherichia coli multicopy plasmids. $\mathrm{Mol}$ Microbiol 63, 35-43.

Chatwin, H. M. \& Summers, D. K. (2001). Monomer-dimer control of the ColE1 $\mathrm{P}_{\text {cer }}$ promoter. Microbiology 147, 3071-3081.

Colloms, S. D., Sykora, P., Szatmari, G. \& Sherratt, D. J. (1990). Recombination at ColE1 cer requires the Escherichia coli xerC gene product, a member of the lambda integrase family. J Bacteriol 172, 6973-6980.

Colloms, S. D., McCulloch, R., Grant, K., Neilson, L. \& Sherratt, D. J. (1996). Xer-mediated site-specific recombination in vitro. EMBO J 15, $1172-1181$
Finkel, S. E. \& Johnson, R. C. (1992). The FIS protein: it's not just for DNA inversion any more. Mol Microbiol 6, 3257-3265.

Gillen, J. R., Willis, D. K. \& Clark, A. J. (1981). Genetic analysis of the RecE pathway of genetic recombination in Escherichia coli K-12. J Bacteriol 145, 521-532.

Guhathakurta, A., Viney, I. \& Summers, D. (1996). Accessory proteins impose site selectivity during ColE1 dimer resolution. Mol Microbiol 20, 613-620.

Hengen, P. N., Bartram, S. L., Stewart, L. E. \& Schneider, T. D. (1997). Information analysis of FIS binding sites. Nucleic Acids Res 25, 4994-5002.

Higuchi, R., Krummel, B. \& Saiki, R. K. (1988). A general method of in vitro preparation and specific mutagenesis of DNA fragments: study of protein and DNA interactions. Nucleic Acids Res 16, 7351-7367.

Ho, S. N., Hunt, H. D., Horton, R. M., Pullen, J. K. \& Pease, L. R. (1989). Site-directed mutagenesis by overlap extension using the polymerase chain reaction. Gene 77, 51-59.

Hodgman, T. C., Griffiths, H. \& Summers, D. K. (1998). Nucleoprotein architecture and ColE1 dimer resolution: an hypothesis. Mol Microbiol 29, 545-558.

Johnson, R. C., Ball, C. A., Pfeffer, D. \& Simon, M. I. (1988). Isolation of the gene encoding the Hin recombinational enhancer binding protein. Proc Natl Acad Sci U S A 85, 3484-3488.

Kennedy, C. K. (1971). Induction of colicin production by high temperature or inhibition of protein synthesis. J Bacteriol 108, 10-19.

Kitagawa, M., Ara, T., Arifuzzaman, M., loka-Nakamichi, T., Inamoto, E., Toyonaga, H. \& Mori, H. (2005). Complete set of ORF clones of Escherichia coli ASKA library (a complete set of E. coli K-12 ORF archive): unique resources for biological research. DNA Res 12, 291-299.

Livak, K. J. \& Schmittgen, T. D. (2001). Analysis of relative gene expression data using real-time quantitative PCR and the $2^{\Delta \Delta C_{\mathrm{T}}}$ method. Methods 25, 402-408.

Muller-Hill, B. (2006). What is life? The paradigm of DNA and protein cooperation at high local concentrations. Mol Microbiol 60, 253-255.

Nordström, K., Ingram, L. C. \& Lundback, A. (1972). Mutations in Rfactors of Escherichia coli causing an increased number of R-factor copies per chromosome. J Bacteriol 110, 562-569.

Patient, M. E. \& Summers, D. K. (1993). ColE1 multimer formation triggers inhibition of E. coli cell division. Mol Microbiol 9, 1089-1095.

Stirling, C. J., Szatmari, G., Stewart, G., Smith, M. C. M. \& Sherratt, D. J. (1988). The arginine repressor is essential for plasmid stabilizing sitespecific recombination at the ColE1 cer locus. EMBO J 7, 4389-4395.

Stirling, C. J., Colloms, S. D., Collins, J. F., Szatmari, G. \& Sherratt, D. J. (1989). xerB, an Escherichia coli gene required for plasmid ColE1 site-specific recombination is identical to $p e p A$, encoding aminopeptidase $A$, a protein with substantial similarity to bovine lens leucine aminopeptidase. EMBO J 8, 1623-1627.

Strater, N., Sherratt, D. J. \& Colloms, S. D. (1999). X-ray structure of aminopeptidase A from Escherichia coli and a model for the nucleoprotein complex in Xer site-specific recombination. EMBO J 18, 4513-4522.

Summers, D. K. \& Sherratt, D. J. (1984). Multimerization of high copy number plasmids causes instability: ColE1 encodes a determinant essential for plasmid monomerization and stability. Cell 36, 1097-1103.

Summers, D. K., Beton, C. W. H. \& Withers, H. L. (1993). Multicopy plasmid instability: the dimer catastrophe hypothesis. Mol Microbiol 8, 1031-1038.

Vieira, J. \& Messing, J. (1982). The pUC plasmids, an M13mp7derived system for insertion mutagenesis and sequencing with synthetic universal primers. Gene 19, 259-268.

Edited by: L. S. Frost 